\title{
Application of Thermosensitive Peptide Copolymer Gels to Removal of Endocrine Disruptor
}

\author{
Satoshi Tanimoto, Naoto Yagi, and Hitoshi Yamaoka \\ Department of Materials Science, The University of Shiga Prefecture, Hassaka, Hikone 522-8533, Japan \\ Correspondence should be addressed to Satoshi Tanimoto, tanimoto@mat.usp.ac.jp \\ Received 22 April 2009; Accepted 2 July 2009 \\ Recommended by Peter Majewski
}

Poly(L-leucine)-block-poly(ethylene glycol)-block-poly(L-leucine) triblock copolymers were synthesized by a ring-opening polymerization of $\alpha$-amino acid $\mathrm{N}$-carboxyanhydride with amino-terminated PEG as an initiator. The chloroform solution of these peptide copolymers showed a thermo-sensitive sol-gel transition. The transition temperature varied as a function of the length of peptide segments. Additionally, we used these peptide copolymers to remove an endocrine disruptor such as bisphenol A from its aqueous solution. As a result, it became clear that the peptide copolymer gel used in this study could capture bisphenol A efficiently.

Copyright (C) 2009 Satoshi Tanimoto et al. This is an open access article distributed under the Creative Commons Attribution License, which permits unrestricted use, distribution, and reproduction in any medium, provided the original work is properly cited.

\section{Introduction}

Since the ring-opening polymerization of $\alpha$-amino acid $\mathrm{N}$ carboxyanhydride was widely introduced by Kricheldorf [1, 2], various type of peptide (poly(amino acid)) has been synthesized in the world. The history of polymerization of $\alpha$ amino acid N-carboxyanhydride was described in Deming's article in detail [3]. Recently, block copolymers that contain peptide segments are studied eagerly in the various fields. For example, the copolymer which consists of two kinds of poly (amino acid) is being researched for the purpose of developing stimulus responsible materials [4].

Poly(ethylene glycol) (PEG) is known to have a low toxicity and not to produce an antibody after the injection into human body. Additionally, it is biocompatible and appropriate for using in vivo [5]. Hence, the block copolymer containing PEG segments have recently been studied as a biofunctional material, and the molecular design of them has been examined energetically [6-10].

In this study, we synthesized the block copolymer of PEG of which poly(L-leucine) combined with both ends and did the removal experiment of the organic molecules from their water solutions by using organogels of this copolymer. As a removal object, we chose an endocrine disruptor such as bisphenol A. Endocrine disruptors are thought to influence generative functions, and therefore it is an urgent subject to remove them from the environment [11-13].

\section{Experimental}

2.1. Materials. L-Leucine and bis(trichloromethyl)carbonate (triphosgene) were purchased from Wako Pure Chemicals Ltd., Tokyo, Japan and used without further purification. $\mathrm{O}, \mathrm{O}^{\prime}$-Bis (2-aminoethyl)poly(ethylene glycol) (amino-PEG, molecular weight: 1500 and 3000) was purchased from Fluka Chemie GmbH, Switzerland and used after precipitation into 100-fold diethyl ether from chloroform solution at room temperature. THF, DMF, n-hexane, diethyl ether, and chloroform were purchased from Wako Pure Chemicals Ltd., Tokyo, Japan and used after distillation by appropriate methods.

2.2. Synthesis of poly(L-leucine)-block-poly(ethylene glycol)block-poly(L-leucine) (PLL-b-PEG-b-PLL). Synthesis of Lleucine N-carboxyanhydride (Leu-NCA) was carried out by the Fuchs-Farthing method using triphosgene. Triphosgene was dissolved in THF and added to L-leucine suspension in THF. The mixture was stirred at $323 \mathrm{~K}$. After the conversion to Leu-NCA was finished, the solvent was removed under reduced pressure. The obtained Leu-NCA was recrystallized 


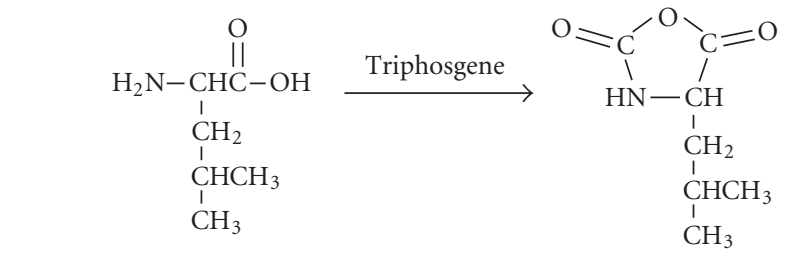

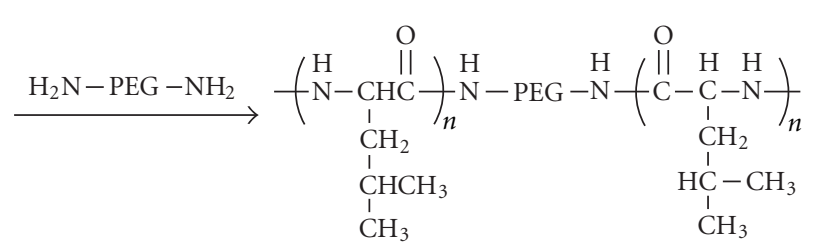

Scheme 1: Schematic diagram of monomer synthesis and ringopening polymerization.

three times from a mixture of THF/n-hexane and was dried by a freeze-drying procedure. Amino-PEG was added to the DMF solution of Leu-NCA as an initiator under nitrogen atmosphere. The completion of the polymerization was evaluated by IR spectrum. The resulting product was precipitated into diethyl ether from chloroform solution. The synthesis procedure is summarized in Scheme 1.

2.3. Characterization of Synthesized Peptide Copolymers. The polymerization degree was determined by $400 \mathrm{MHz}{ }^{1} \mathrm{H}-$ NMR (JEOL, JMN-LA400) in chloroform-d. Gel permeation chromatography (GPC) measurement was carried out at $313 \mathrm{~K}$ in chloroform (flow rate: $1.0 \mathrm{~mL} / \mathrm{min}$ ) using liquid chromatograph system purchased from Shimadzu Corporation, Japan. The molecular weight and polydispersity ratio $(\mathrm{Mw} / \mathrm{Mn})$ of the polymers were calculated from chromatographs relative to commercial polystyrene standards. Differential scanning calorimetry (DSC) measurement was performed from $313 \mathrm{~K}$ to $273 \mathrm{~K}$ (cooling speed: $2 \mathrm{~K} / \mathrm{min}$ ) using DSC-8230 (Rigaku, Japan).

2.4. Sol-Gel Transition. The sol-gel transition was observed by swinging temperature of chloroform solution containing a peptide copolymer. Turbidity measurement was difficult because the peptide copolymer solution in chloroform was transparent before and after gelation.

2.5. Removal Experiment of Endocrine Disruptor. We did the removal experiment of an endocrine disruptor in a very simple way, which consisted of a temperature control and a stirring. We chose bisphenol $\mathrm{A}$ as a representative of the endocrine disrupter. First, we made an aqueous solution saturated with the objective material (in this study, bisphenol A) in the test tube. We added the chloroform solution of the peptide copolymer into the aqueous solution of bisphenol A. We stirred the mixture by a magnetic stirrer with holding temperature at $313 \mathrm{~K}$ (above the sol-gel transition temperature). After stirring it for 1 minute, we stopped
TABLE 1: Characterization of synthesized peptide copolymer. Mn, $\mathrm{Mw}$, and Mw/Mn were obtained from GPC measurement. Polymerization degrees were calculated from NMR spectrum.

\begin{tabular}{lcccc}
\hline Notation & Mn & Mw & Mw/Mn & PLL : PEG $:$ PLL (units) \\
\hline I & 3987 & 5189 & 1.30 & $5: 66: 5$ \\
II & 5502 & 8038 & 1.46 & $7: 66: 7$ \\
III & 6939 & 10224 & 1.47 & $10: 66: 10$ \\
IV & 1064 & 2028 & 1.91 & $6: 31: 6$ \\
V & 3532 & 5073 & 1.44 & $11: 31: 11$ \\
VI & 3520 & 7097 & 2.02 & $14: 31: 14$ \\
\hline
\end{tabular}

stirring and left it quietly at $313 \mathrm{~K}$ until the boundary became clear. The solubility of the copolymer in water was small enough due to the hydrophobicity of the polyleucine chain, and the copolymer could not move from chloroform phase to water phase. Consequently, the aqueous phase was above the boundary, and under the boundary was the chloroform solution of the copolymer. Next, we cooled down the whole system to the temperature at which gelation occurred with taking care of not disturbing the boundary. After the lower layer gelled, we only slanted the test tube and could take out an upper layer (aqueous phase). We were able to confirm the concentration of bisphenol $\mathrm{A}$ in the aqueous phase from the absorbance at $277 \mathrm{~nm}$ obtained by UV spectrophotometer.

\section{Results and Discussion}

3.1. Characteristic Properties of Peptide Copolymer Synthesized in This Study. Amino-PEG was used as an initiator for NCA ring-opening polymerization. The molecular weights of amino-PEGs used in this study were 1500 and 3000. Terminal amino groups of the amino-PEG attacked the carbonyl carbon of NCA-rings, and the polymerization proceeded like a living polymerization. As a result of GPC measurement, the molecular weight distribution was narrow enough, and $\mathrm{Mw} / \mathrm{Mn}$ evaluated by GPC was shown in Table 1 . From the result of NMR measurement, the number of leucine units combined at each end of PEG chain was determined. The NMR results showed a good agreement with the results of GPC measurement. The whole results of characterization are summarized in Table 1.

3.2. Thermosensitive Sol-Gel Transition. The solution at $313 \mathrm{~K}$ was seen as a clear liquid. This solution showed quick gelation without turbidity change when it was cooled down to the room temperature. This transition could be seen in all copolymers. Figure 1 shows that the transition temperature depended on the length of the peptide segment and on the concentration of chloroform solution of the peptide copolymer. We investigated thermal nature of the copolymer itself and of its chloroform solution by using differential scanning calorimetry, and an exothermic peak related to sol-gel transition was observed (not shown here). As a result of observations of copolymers with various length of peptide, it was observed that the transition temperature shifted to higher temperature with increasing the length of 


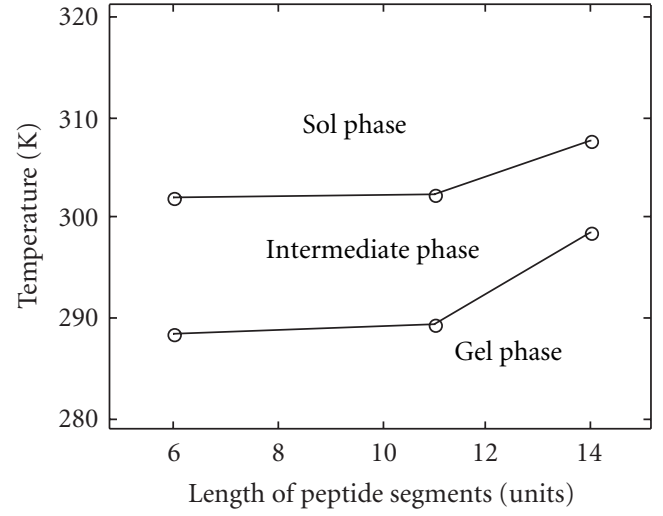

(a)

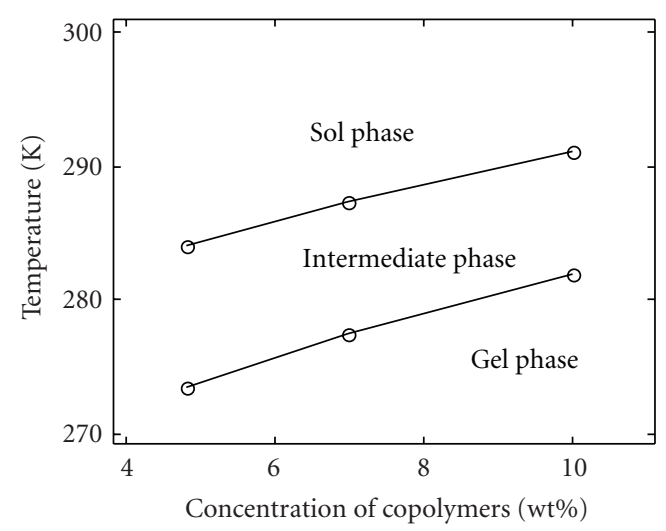

(b)

FIGURE 1: Sol-gel transition temperature of chloroform solution of copolymer IV, V, and VI. (a) shows a peptide length dependence, and (b) shows a copolymer concentration dependence.

poly(L-leucine) segments. The increase in sol-gel transition temperature was common in our whole observation though the increase between $n=6$ and $n=11$ was a little. It suggests that the gelation of peptide copolymer solution was induced by entanglement of peptide segments. Because the hydrophobic interaction among polyleucine chains causes the entanglement of the peptide segments, which forms a junction point of the gel, the longer peptide chain causes tighter hydrophobic binding and raises the sol-gel transition temperature more rapidly.

\subsection{Application to Removal Process of Endocrine Disruptors} from Their Aqueous Solution. We did the removal experiment of the bisphenol A by the process described in Section 2. In Figure 2, we showed the results of the removal experiment in the case of bisphenol A. After we applied the removal procedure to the saturated solution of bisphenol A, the concentration decreased to less than $1 / 6$. Then, we repeated the same operation and evaluated a decrease in concentration of the bisphenol A after each cycle. After we repeated this process three times, the concentration of the bisphenol A decreased, and we could not detect it any more with UV spectroscopy. As a control experiment, we

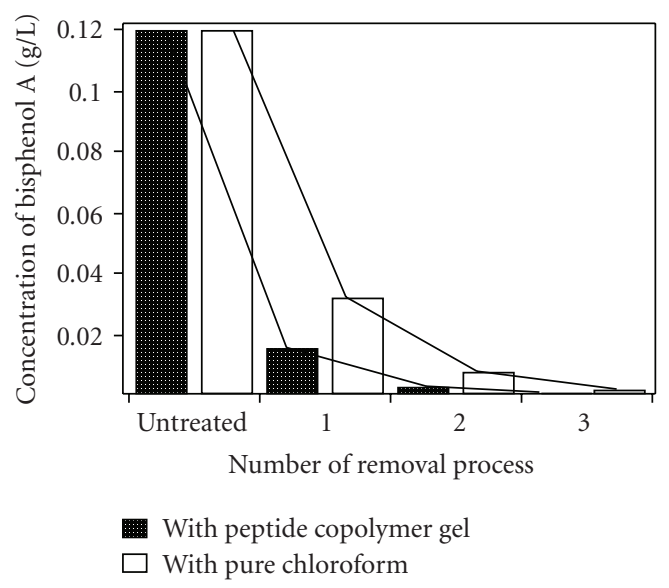

FIgure 2: Results of removal experiments. The shaded rectangle shows the result of a trial with peptide copolymer gel, while the open rectangle corresponds to the result of an extraction with pure chloroform.

operated an extraction by using the chloroform of the same quantity and compared a decrease in concentration. As a result, it became clear that the scavenging process with peptide copolymer gel could remove more bisphenol A than the extraction process only with chloroform. Moreover, in the case of the method carried out without gelation, the separation between aqueous phase and chloroform phase was so difficult that the resulting removal efficiency was similar to the value of the extraction with chloroform. As for other aromatic molecules as well, we tried the same process again. The decrease in concentration showed the similar behavior to the case of bisphenol A. These results suggest that bisphenol A was held in gel matrix due to the gelation of the peptide copolymer. Though we are investigating the detail of capturing mechanism now, we consider that the molecule of bisphenol A is mainly captured by a hydrophobic interaction, and the hydrophobicity of leucine segment is a driving force of gelation.

\section{Conclusion}

Peptide copolymers containing PEG and poly(L-leucine) chains were synthesized by a ring-opening polymerization of $\alpha$-amino acid $\mathrm{N}$-carboxyanhydride. The chloroform solution of peptide copolymer synthesized in this study showed a thermosensitive sol-gel transition. And it became clear that the peptide copolymer gel can be used to scavenge bisphenol A from its aqueous solution efficiently with simple three steps (stirring, cooling, and decantation). This process proposed in this study will be applied to the environmentally harmonized water treatment in the near future.

\section{Acknowledgment}

This study was supported in part by a grant for "The Development of Base Technology for the Construction of Environmentally Harmonious Industrial Systems" promoted 
by Shiga Prefecture under the program of Collaboration of Regional Entities for the Advancement of Technological Excellence by Japan Science and Technology Agency.

\section{References}

[1] H. R. Kricheldorf, $\alpha$-Aminoacid-N-Carboxyanhydrides and Related Materials, Springer, New York, NY, USA, 1987.

[2] H. R. Krisheldorf, in Models of Biopolymers by Ring-Opening Polymerization, S. Penczek, Ed., CRC, Boca Raton, Fla, USA, 1990.

[3] T. J. Deming, "Living polymerization of $\alpha$-amino acid-Ncarboxyanhydrides," Journal of Polymer Science: Part A, vol. 38, no. 17, pp. 3011-3018, 2000.

[4] A. P. Nowak, V. Breedveld, L. Pakstis, et al., "Rapidly recovering hydrogel scaffolds from self-assembling diblock copolypeptide amphiphiles," Nature, vol. 417, no. 6887, pp. 424-428, 2002.

[5] I. N. Topchieva, N. V. Efremova, N. V. Khvorov, and N. N. Magretova, "Synthesis and physicochemical properties of protein conjugates with water-soluble poly(alkylene oxides)," Bioconjugate Chemistry, vol. 6, no. 4, pp. 380-388, 1995.

[6] A. Harada, S. Cammas, and K. Kataoka, "Stabilized $\alpha$-helix structure of poly(L-lysine)-block-poly(ethylene glycol) in aqueous medium through supramolecular assembly," Macromolecules, vol. 29, no. 19, pp. 6183-6188, 1996.

[7] K. Emoto, M. Iijima, Y. Nagasaki, and K. Kataoka, "Functionality of polymeric micelle hydrogels with organized threedimensional architecture on surfaces," Journal of the American Chemical Society, vol. 122, no. 11, pp. 2653-2654, 2000.

[8] T. Doi, T. Kinoshita, Y. Tsujita, and H. Yoshimizu, "Control of molecular orientation of $\alpha$-helix in the monolayer at air-water interface," Bulletin of the Chemical Society of Japan, vol. 74, no. 3, pp. 421-425, 2001.

[9] A. Harada and K. Kataoka, "Polyion complex micelles with core-shell structure: their physicochemical properties and utilities as functional materials," Macromolecular Symposia, vol. 172, pp. 1-9, 2001.

[10] T. Ishii, H. Otsuka, K. Kataoka, and Y. Nagasaki, "Preparation of functionally PEGylated gold nanoparticles with narrow distribution through autoreduction of auric cation by $\alpha$-Biotinyl-PEG-block-[poly(2-(N,N-dimethylamino)ethyl methacrylate)]," Langmuir, vol. 20, no. 3, pp. 561-564, 2004.

[11] S. C. Nagel, F. S. Vom Saal, K. A. Thayer, M. G. Dhar, M. Boechler, and W. V. Welshons, "Relative binding affinityserum modified access (RBA-SMA) assay predicts the relative in vivo bioactivity of the xenoestrogens bisphenol A and octylphenol," Environmental Health Perspectives, vol. 105, no. 1, pp. 70-76, 1997.

[12] F. S. vom Saal, P. S. Cooke, D. L. Buchanan, et al., "A physiologically based approach to the study of bisphenol a and other estrogenic chemicals on the size of reproductive organs, daily sperm production, and behavior," Toxicology and Industrial Health, vol. 14, no. 1-2, pp. 239-260, 1998.

[13] M. Wagner and J. Oehlmann, "Endocrine disruptors in bottled mineral water: total estrogenic burden and migration from plastic bottles," Environmental Science and Pollution Research, vol. 16, no. 3, pp. 278-286, 2009. 

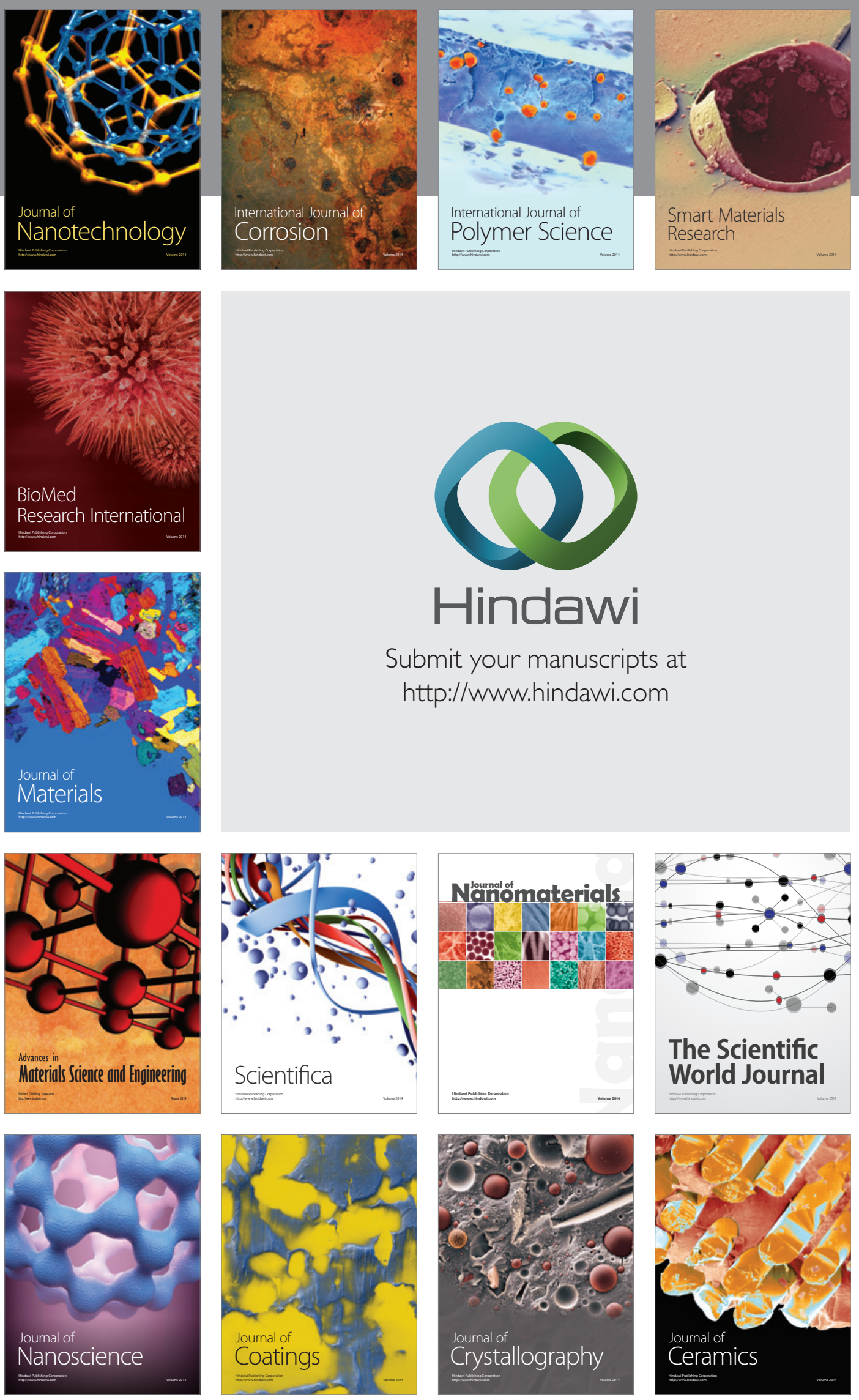

The Scientific World Journal

Submit your manuscripts at

http://www.hindawi.com

\section{World Journal}

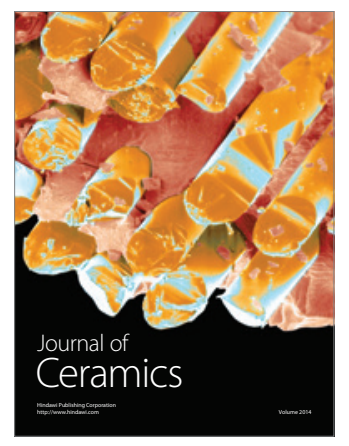

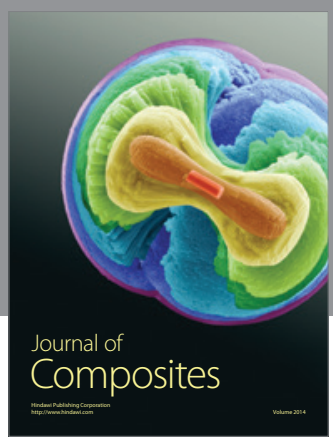
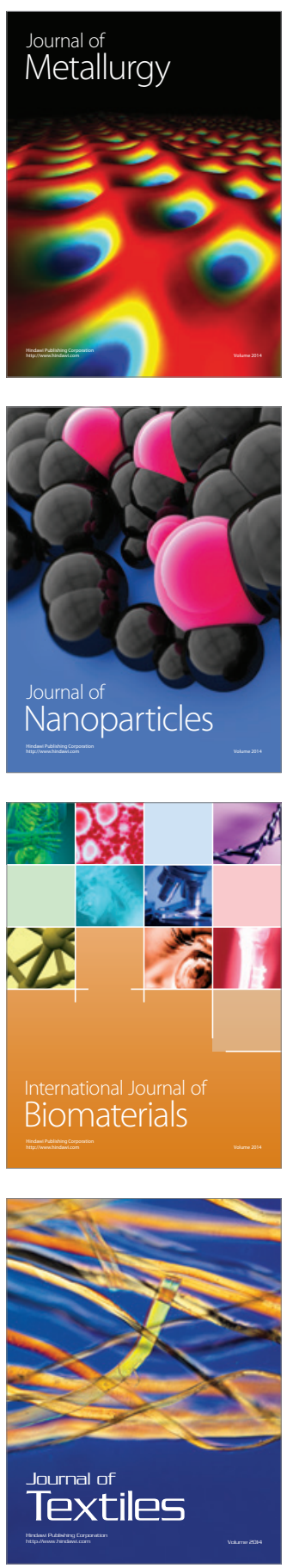